\title{
PRONOMI, AVVERBI E PREPOSIZIONI
}

"Bruciava già quando mi ci sono sdraiato" è il titolo della traduzione italiana di un libro umoristico americano, nell'originale When I Lay Down on I $t^{1}$. Ricostruendo la frase intera in entrambe le lingue (si tratta, a quanto pare, delle parole di un uomo che spiega come si è sviluppato l'incendio in casa sua) otteniamo:

( 1) Il letto bruciava già quando mi ci sono sdraiato

( 2) The bed was already burning when I lay down on it

dove il pronome it sostituisce (o "rappresenta", o "pronominalizza", a seconda della concezione dei pronomi da cui si vuole partire) il sintagma the bed. È chiaro che anche in italiano, partendo dalla struttura di base

( 3) Il letto bruciava già quando mi sono sdraiato sul letto

si potrebbe avere una sostituzione analoga a quella dell'inglese, cioè

( 4) Il letto bruciava già quando mi sono sdraiato su (di) esso

La frase è certamente grammaticale, ma alquanto pesante. Perciò l'italiano preferisce eseguire una seconda sostituzione, "condensando" ${ }^{\text {2 }}$ il sintagma preposizionale in ci e ottenendo (1).

Conclusioni quasi identiche valgono per il secondo periodo dell'esempio

( 5) Ti piace la tua pelliccia? Il suo proprietario $c$ 'è stato ammazzato dentro! ${ }^{3}$ che tradotta in inglese suonerebbe:

( 6) (Do you like your fur coat?) Its owner has been murdered in it!

Ancora una volta, lo stesso costrutto potrebbe valere anche per l'italiano come struttura sottostante ("Il suo proprietario è stato ammazzato in essa"), ma per spie-

1 Di Robert Fulghum, ed. Frassinelli, Milano 1990.

2 Per questo termine si veda R. Simone, Punti di attacco dei clitici in italiano, in: Italia linguistica: idee, storia, strutture, a cura di F. Albano Leoni (e altri), Bologna 1983, 285-307, in particolare a p. 290.

3 Manifesto della Lega anti-vivisezione, citato in Panorama 9-XII-1990, p. 67. 
gare la comparsa della parola dentro è necessario presupporre "ammazzato dentro di essa", o addirittura un (bruttissimo, ma indispensabile per la derivazione di $c i$ ) "dentro ad essa".

Costrutti analoghi, con $c i / v i$ e parole definite come "avverbi" nella grammatica tradizionale, si hanno negli esempi seguenti:

( 7) Egli rende soffice il terreno con uno strato di foglie, vi stende sopra dei ramoscelli...

( 8) C'era Giovanni, e mi ci sono seduto vicino.

(9) Ma perché mi ci avete messo assieme?

Oltre a $c i / v i$, compaiono nello stesso ruolo (logicamente, poiché anch'essi, come $c i / v i$ nella maggior parte dei loro impieghi, sostituiscono un sintagma $a+$ sostantivo) i pronomi atoni dativali:

(10) Tutta colpa della campagna che $c i$ hanno scatenato contro.

(11) Non c'è stato nessuno che abbia patito, agonizzato più di lei. Ma tuttỉ le sono stati contro, perché non sopportano il suo male.

(12) Tra Cannero e la foce del Tresa mi si aprivano davanti le acque di casa.

(13) L'Orimbelli la fece accomodare sulla panchetta e le sedette di fronte.

(14) Non amiamo più Ciriaco come una volta, ma non gli stiamo di certo addosso col fucile puntato.

(15) Il nostro cane alano Molok $t i$ è corso addosso abbaiando. ${ }^{5}$

La combinazione di un clitico con "avverbi“ come dentro, sopra, davanti, di fronte, contro, addosso etc. è un procedimento molto frequente nella sintassi italiana, sul quale però invano cercheremmo il minimo cenno nelle grammatiche tradizionali. Ora finalmente ne dà un'analisi particolareggiata Luigi Rizzi nella Grande grammatica a cura di L. Renzi . Evitando di parlare di "avverbi" (persino in esempi

4 Il primo esempio è di Italo Calvino, Lezioni americane, Milano 1988, p. 7; gli altri due si trovano in M. Berretta, "Ci"vs. "gli": un microsistema in crisi?, nel volume della SLI, Sintassi e morfologia della lingua italiana d'uso, a cura di A. Franchi de Bellis e L.M. Savoia, Roma 1985, a p. 125.

5 Gli esempi provengono: (10) dalla stampa, citato nella Grammatica italiana di L. Serianni, Torino 1988, p. 391; (11) da E. Biagi, Amori, Milano 1988, p.78; (12) e (13) da P. Chiara, La stanza del vescovo, Milano 1984, p. 78 e 173; (14) da G. Pansa, Carte false, Milano 1988, 260; (15) da A. Moravia, Il dio Kurt, Milano 1968, p. 53.

- Grande grammatica italiana di consultazione, a cura di Lorenzo Renzi, Bologna 1989, capitolo 10 (spec. alle pp. 521-530). 
quali Vado su o Gianni abita accanto egli vede soltanto "preposizioni senza complementi"), il Rizzi chiama "preposizioni polisillabiche" le tradizionali preposizioni "improprie" e le divide in tre classi: (I) quelle che, per formare un sintagma preposizionale, richiedono l'aggiunta di a (accanto, addosso, davanti, incontro, intorno ecc.) o di qualche altra preposizione (invece di, prima di, fuori di/da, lontano da, insieme con); (II) quelle per cui l'aggiunta di $a$ è facoltativa: dietro, contro, dentro, sopra, sotto ecc. (p.es. dietro la porta = dietro alla porta); (III) quelle che non ammettono una seconda preposizione: dopo, verso, circa, senza, durante, malgrado, secondo ecc. Queste ultime ovviamente non sono interessanti per il nostro argomento (con la sola eccezione di senza, che può apparire in costrutti come "Ne sono rimasto senza“: perciò lo stesso autore, a p. 526, considera la possibilità di inserire senza piuttosto nella classe II come caso speciale, cioè con l'aggiunta di un di cancellato in superficie anziché di un $a$ ).

Commentando esempi come

(16) Gianni ci si è messo dietro (alla porta)

(17) Ci sto accanto

(18) Gianni gli si è messo dietro (a Mario)

(19) Mario le gira intorno

(20) Le vivo accanto

e altri, evidentemente analoghi ai nostri esempi (5)-(15), insieme con quelli dove al posto di ci o pronome dativale si ha la preposizione $a$ con un pronome relativo o interrogativo:

(21) La ragazza alla quale sono andato incontro...

(22) La porta a cui mi son messo dietro...

(23) La ragazza alla quale Gianni si è messo accanto...

(24) A chi ti sei messo accanto?

- il Rizzi parla di "estrazione del sintagma preposizionale interno" e constata che questa vale quasi sempre per le "preposizioni polisillabiche" combinate con $a$, a parte le combinazioni insieme con e fuori da (di, invece, "non sembra estraibile"), come in:

(25) $\mathrm{Ci}$ vivo insieme (con Maria) 
(26) La persona con la/alla quale vivo insieme...

(27) Ne sono finalmente fuori (da questo incubo)

(28) Questo è l'incubo da cui sono finalmente fuori.

Chiara, precisa e ricca di informazioni nuove, la trattazione di Rizzi tuttavia ha dovuto lasciare, in una materia così complessa, non pochi contorni sfumati e domande senza una risposta precisa. Oltre al già nominato senza, è il caso di su, monosillabo che da una parte (p.es. in il libro sul tavolo) è una preposizione comune, nemmeno "impropria“; dall'altra appare in costrutti come

(29) $C i$ devo pensare su

(30) Dormici su

che sembrano paragonabili agli altri esempi di sopra. Il Rizzi, però (p. 527-528), osservando che si tratta di "una forma idiomatica limitata ad un ristretto numero di significati e scelte verbali“ e che non è accettabile ad es. *Questa storia, alla quale devo pensare su..., rifiuta di ammettere anche questo tra i casi di "estrazione“. Ora, se pensiamo all'evidente parallelismo, sincronico e diacronico, tra su e giù (indubbiamente avverbio quest'ultimo), pare che la soluzione migliore sia di ammettere l'esistenza di due su, uno preposizionale, l'altro avverbiale (distinti anche foneticamente, essendo l'uno proclitico e tonico l'altro) ${ }^{7}$. Questo a sua volta ci induce a cercare una descrizione più equilibrata per l'intera categoria di parole suscettibili di "estrazione". Non sono avverbi veri e propri, secondo la definizione troppo semplicistica delle grammatiche scolastiche e dei dizionari, ma nemmeno il termine "preposizione polisillabica " sembra soddisfacente. Piuttosto, come tante volte nell'analisi grammaticale, abbiamo a che fare con una gradazione. Fra i veri avverbi di luogo da una parte, come qui, là, gì̀ (e anche su), e le preposizioni "proprie" di, a per ecc. dall'altra, le tre classi delimitate da Rizzi costituiscono una fascia intermedia di "avverbi-preposizioni" o "preposizioni-avverbi" che dir si voglia. Le forme della classe I sono ancora sostanzialmente avverbi, tanto da richiedere l'aggiunta di $a$ o altra preposizione "propria" per poter funzionare da preposizioni. In quelle della classe II l'aggiunta, come si è visto, è facoltativa. Quelle della terza classe, infine, differiscono dalle preposizioni "proprie" soltanto per il fatto puramente fonetico di avere più d'una sillaba e per la possibilità che hanno alcune di esse (non tutte e certamente non in tutti gli usi) di collocarsi in fine di sintagma (tre ore dopo, fare senza, mio malgrado ecc.).

Se ora però ci domandiamo quale sia la ragion d'essere dell'“estrazione“, quale funzione essa svolga nel repertorio delle strutture sintattiche italiane, arriveremo al-

7 Il parallelismo si estende alle coppie di avverbi composti lassù - laggiù e quassù - quaggiù. Il Rizzi, mentre riconosce (p. 530) che in laggiù e quaggiù "il modificatore non è preposizionale", non menziona affatto lassù e quassù. 
la conclusione che l'uso delle preposizioni-avverbi non può essere esaminato se non in stretta interdipendenza con i pronomi. Nel caso dei pronomi relativi e interrogativi, lo spostamento dell'avverbio-preposizione consente di ricorrere anche in questi casi (p.es. nelle frasi $21-24$ di sopra) al nesso abituale $a+$ pronome. Senza l' 'estrazione" si otterrebbero domande meno espressive, con il pronome interrogativo troppo lontano dall'inizio della frase ("Accanto a chi ti sei messo?"), costrutti relativi pesanti, tipici della lingua scritta ("la ragazza incontro alla quale sono andato", "la ragazza accanto alla quale Gianni si è messo") o addirittura impossibili (*Questo è l'incubo fuori da cui sono finalmente).

La grande maggioranza dei casi di "estrazione", però - come si vede anche dai nostri esempi - riguarda la cliticizzazione, con $c i / v i$, con ne (esempio 27) o con pronomi dativali. Da questo punto di vista "estrazione" non è forse il termine più adatto: il sintagma preposizionale "interno", una volta "estratto" dal nesso con l'avverbio-preposizione, non per questo diventa più visibile, ma al contrario scompare, assorbito nel sostituente atono. L'alternativa alla cliticizzazione è l'uso dei pronomi tonici: si confronti (1) e (4). (La ripetizione del sostantivo antecedente all'interno della stessa proposizione, come in (3), è una possibilità puramente teorica che nel discorso reale non si verifica quasi mai.) La scelta fra "estrazione" e "non estrazione", quindi, si riduce alla scelta fra pronome tonico e pronome atono, ed è ben noto che quest'ultima non è arbitraria, ma è dettata da una serie di fattori semantici e sintattici e dalle esigenze di quello che oggi si chiamerebbe "focalizzazione" o "topicalizzazione". Se il contenuto del messaggio è tale da richiedere il pronome atono, l'"estrazione" avviene come una necessaria conseguenza sintattica.

Prendendo a caso alcuni dei nostri esempi, in (12) il narratore che racconta la sua navigazione solitaria non ha alcun motivo di mettersi in rilievo dicendo "si aprivano davanti a me le acque di casa". In (15), dire "il cane alano è corso addosso $a$ te avrebbe senso solo se alla scena rievocata fossero state presenti altre persone che il cane avrebbe potuto assalire. In (19), dove possiamo immaginare un contesto del tipo "Gianna è una bella ragazza, Mario le gira intorno", l'uso del sintagma preposizionale ("...gira intorno a lei") creerebbe un'enfasi quasi altrettanto ingiustificata come se invece di "... Mario la corteggia" dicessimo "... Mario corteggia lei". Certamente agiscono anche fattori stilistici: in (11), "...ma tutti sono stati contro di lei" avrebbe per conseguenza una sgradevole ripetizione del "di lei“ immediatemente preecedente.

D'altra parte va notato che esiste un'indubbia ripugnanza a usare i nessi di preposizione + pronome in riferimento a inanimati, cioè, in pratica, i nessi con esso, $-a,-i,-e, \mathrm{i}$ quali perciò tendono a cedere il posto, appena possibile, alle particelle ${ }^{8} . \grave{\mathrm{E}}$

8 Di questo fenomeno i linguisti italiani non sembrano essersi occupati finora, a parte un brevissimo cenno di Monica Berretta sulle "terze persone, in cui si affollano più forme per referenti animati, mentre - per la scarsa vitalità nel parlato della serie esso/a/i/e e del neutro 'standard' ciò - mancano forme adeguate per réferenti non animati soggetti e oggetti indiretti" (op. cit., 117). Si veda anche il mio articolo Sulle funzioni attuali del pronome ESSO, "Lingua nostra", 1976, XXXVII, fasc. 1-2, $26-32$. 
per questo che le nostre ricostruzioni proposte all'inizio, "mi sono sdraiato su di esso" (es. 4) e "ammazzato dentro ad essa" hanno un suono pesante e poco naturale, e lo stesso varrebbe per altri esempi in cui si volesse ripristinare prep. + esso al posto di $c i / v i$ :

( 7a) Egli rende soffice il terreno... stende sopra di esso (ad esso) dei ramoscelli...

(16a) Gianni si è messo dietro di essa (ad essa)

(17a) Sto accanto ad esso

ecc. Ci sono però altri casi, specialmente in testi di tono più elevato, dove è proprio la particella che suona male, rasentando a volte l'inaccettabilità, senza che se ne vedano chiaramente le ragioni. Le "restrizioni sull'estrazione" determinate dall Rizzi" sono utili, ma dovrebbero appogiarsi su regole più generali per la sostituibilità di prep. + pronome tonico con pronome atono o particella (dunque anche senza avverbi-preposizioni, come nel nostro esempio 1). Parlare di "regole" forse è pretendere troppo, ma si sente indubbiamente il bisogno di criteri oggettivi per spiegare perché, mentre (1) è senz'altro preferibile a (4), in altri casi scrittori la cui padronanza dell'italiano non può essere messa in dubbio optano proprio per il sintagma su (di) esso, p.es.:

(31) Io non ho lo minima idea del perché [mia moglie] non possa andare con quel vestito. Ma non insisto su esso.

(32) Piegò le dita a uncino e inarcò il palmo della mano posandoli sul sasso sino a far presa su di esso.

(33) La forma sé si dice "riflessiva“, perché a differenza di lui, lei, loro si riferisce allo stesso soggetto (cioè si riflette su di esso...) ${ }^{10}$

Quando si cercherà una risposta a queste difficili domande, sarà bene farlo in una prospettiva "interlinguistica". Infatti l'italiano, tra le lingue europee, è proba-

9 Op. cit., 526-527. Il Rizzi constata che il sintagma preposizionale interno è estraibile dal sintagma complesso quando quest'ultimo è complemento del verbo; se è avverbiale del sintagma verbale l'estrazione diventa problematica, se è avverbiale di frase l'estrazione è impossibile. Beninteso, come sempre in questioni di grammaticalità e accettabilità, $i$ limiti sono piuttosto vaghi. Cosi l'autore segna come anomala la frase Gianni le ha mangiato accanto e molto anomala La ragazza alla quale Gianni ha mangiato accanto..., entrambe a causa del verbo mangiare (in Gianni le si è messo accanto e La ragazza alla quale Gianni si è messo accanto..., dove accanto è complemento del verbo, l'estrazione è perfettamente accettabile). Sarebbe interessante fare un confronto con il seguente esempio: "Aveva derubato Iddio del suo segreto, d'Iddio era diventato socio, da quel momento gli avrebbe mangiato e dormito insieme" (L. Santucci, Lo zio prete, Milano 1979, p. 34).

10 Il primo esempio è di Achille Campanile, Gli asparagi e l'immortalità dell'anima, Milano 1989, 124; il secondo di Umberto Eco, Diario minimo, Milano 1988, p. 49. Il terzo, della Grammatica italiana descrittiva di M. Regula e J. Jernej, Berna 1975, 143, è tanto più curioso in quanto si trova nella stessa pagina dove gli autori danno un elenco dei pronomi tonici in funzione di complemento - dimenticando (come del resto Battaglia-Pernicone e quasi tutti i grammatici italiani di una volta) di includervi esso, $-a,-i,-e$ ! 
bilmente quella più propensa alla cliticizzazione, grazie all'esistenza delle particelle $c i / v i$ e ne e al largo uso che fa dei pronomi atoni. Nell'inglese, che non ha pronomi clitici, tutte le frasi citate sopra avrebbero un sintagma preposizionale, senza distinzione fra quelle che in italiano hanno l'avverbio-preposizione, come (6), e quelle che ne sono prive, come (2). Qualche esempio:

( 8b) Giovanni was there, and I sat down near him.

(10b) All because of the campaign they have stirred up against us.

(12b) Between Cannero and the mouth of the Tresa, home waters were opening up before me.

(16b) Gianni got behind it

(27b) I'm finally out of it,

e via di seguito. Il serbocroato ha pronomi personali tonici e atoni esattamente come l'italiano, ma non ha particelle equivalenti a $c i / v i$, e d'altro lato fa quasi sempre una netta distinzione tra avverbi e preposizioni. Perciò anche qui, traducendo, otterremo sintagmi preposizionali:

( 1c) Krevet je već goreo kad sam legao na njega.

( 5c) (Svidja li ti se tvoje krzno?) Njegov vlasnik je ubijen $u$ njemu!

(10c) Za sve je kriva kampanja koju su pokrenuli protiv nas.

(12c) Izmedju Cannera i ušća Trese, preda mnom su se otvarale zavičajne vode.

(16c) Gianni je stao iza njih,

eccetera. Solo in pochi casi si hanno avverbi che fungono anche da preposizioni, quali $u$ susret (incontro) o blizu (vicino), e allora diventa possibile l'“estrazione" come in italiano:

(21c) Devojka kojoj sam pošao u susret... (La ragazza alla quale sono andato incontro...)

(34 ) Pošao sam joj u susret (Le sono andato incontro)

( 8c) Bio je tamo Giovanni, pa sam $m u$ seo blizu (C'era Giovanni, e mi ci sono seduto vicino) 
Questi ultimi sono anche gli unici casi in cui si abbia la scelta fra pronome atono e pronome tonico:

(34') Pošao sam u susret njoj (Sono andato incontro a lei)

(8c') Bio je tamo Giovanni, pa sam seo blizu njega (C'era Giovanni, e mi sono seduto vicino a lui)

Per il resto, dato che (come in italiano) soltanto i pronomi tonici possono essere retti da preposizioni, l'opposizione tonico/atono in serbocroato può dirsi neutralizzata. È un tipo di neutralizzazione che anche l'italiano pratica in altri contesti sintattici, necessariamente, in quanto i pronomi atoni e le particelle hanno un campo di applicazione assai più ristretto che non i sintagmi preposizionali. Cosi il contrasto che si ha tra Nessuno se ne ricorda e Nessuno si ricorda di lui viene meno in prima e seconda persona (Nessuno si ricorda di me, Nessuno si ricorda di te); quello tra La formula vi è nota e La formula è nota a voi non è possibile in La formula a voi nota $\grave{e} . .$. , e similmente in molti altri casi. Se ne deduce che la cliticizzazione è ad ogni modo un procedimento parziale, una "seconda sostituzione", anche se in italiano ha possibilità d'impiego più larghe al confronto delle altre lingue.

L'inglese e il serbocroato, non disponendo di alternative all'uso dei sintagmi preposizionali, trattano i pronomi allo stesso modo sia che questi si riferiscano ad animati o a inanimati. In due altre lingue, invece, il francese e il tedesco, questa distinzione è molto più marcata che non in italiano. Il francese si serve degli avverbi ${ }^{11}$ dedans, dehors, dessus, dessous, devant, derrière, autour, ensemble, à côté ecc. Questi possono combinarsi con gli equivalenti delle particelle italiane, $y$ e en rispettivamente (p.es. "Ne sono fuori" = "J'en suis dehors"), ma generalmente ne fanno a meno: così (5) si tradurrebbe "Son propriétaire a été tué dedans" e (16) "Gianni s'est mis derrière". La "coesione", in questo caso, è affidata alla sola vicinanza tra avverbio e antecedente, senza speciali morfemi anaforici. La tonicità è ridotta allo zero: in francese gli inanimati non "meritano", per così dire, nemmeno una particella atona, né esiste la possibilità di indicarli con un sintagma preposizionale come in italiano (mancando qualsiasi pronome corrispondente all'it. esso).

In tedesco, per gli inanimati, il sintagma preposizionale viene sostituito dai "Pronominaladverbien" dafür, dabei, damit, darauf, davon, dazu, dahinter, darüber, ecc. ecc. (più altre due serie: hierfür, hierbei, hiermit, ecc., indicanti la vicinanza, e wofür, wobei, womit, ecc., usati nelle proposizioni interrogative e relative) ${ }^{12}$.

11 Alcuni sono anche preposizioni, come devant e derrière, ma nella maggioranza sono solo avverbi (gli impieghi preposizionali, dove esistono, sono per lo più antiquati). D'altro canto, è noto che nella lingua parlata la preposizione avec si può usare senza complemento ("Que fais-tu de cette brosse? - Je me lave avec"). L'italiano, avendo in questo caso la preposizione proclitica con, può scegliere tra il sintagma preposizionale ("Mi lavo con essa") e la particella ci ("Mi ci lavo").

12 Nell'uso colloquiale, e d'altra parte nella lingua letteraria del passato, si trovano anche sintagmi preposizionali riferiti a inanimati: v. la Duden-Grammatik der deutschen Gegenwartssprache, Mannheim, 1984, pp. 357-358. 
Pur avendo impieghi molto vari, alcune di queste forme sono confrontabili ai clitici italiani ("...quando mi ci sono sdraiato" = "...als ich mich darauf legte") o alla loro combinazione con la preposizione-avverbio ("C"è stato ammazzato dentro" = "Er ist darin getötet worden"). Il morfema $d a(r)$-, in questo caso, effettua la funzione anaforica che in francese manca di espressione formale e che in italiano è affidata al clitico. I "Pronominaladverbien" riuniscono in sé tutt'e tre le parti del discorso nominate nel nostro titolo, in quanto sono composti da un avverbio di luogo e una preposizione, nella frase svolgono funzione avverbiale, sono anaforici come i pronomi, equivalenti del sintagma preposizione + pronome (che verrebbe usato per un referente animato, nella stessa maniera dell'inglese o del serbocroato: "mi ci sono seduto vicino" = "ich setzte mich neben ihn"). Questi mezzi diversi di cui si serve ciascuna lingua per conseguire identici scopi espressivi confermano che l'interdipendenza tra i pronomi e le due categorie di parole invariabili è una proprietà, se non universale, almeno generale nelle lingue europee, basata su un determinato tipo di "struttura profonda" di cui l'analisi sintattica non potrà non tenere conto.

\section{Rezime}

\section{ZAMENICE, PRILOZI I PREDLOZI}

Strukture kao Il suo proprietario c'è stato ammazzato dentro "Njegov vlasnik je ubijen u njemu“ ili Mi si aprivano davanti le acque di casa "Preda mnom su se otvarale zavičajne vode", tj. kombinacije nenaglašene zamenice ili čestice s prilogom-predlogom kao što je dentro, davanti, contro, accanto itd., mada veoma česte u italijanskom, nisu opisane u tradicionalnim gramatikama. One su očigledno izvedene iz oblika sa složenom predloškom sintagmom: (ammazzato) dentro ad essa, (si aprivano) davanti a me. L. Rizzi, koji je prvi analizirao ovakve strukture, prikazuje ih kao rezultat "ekstrakcije" unutrašnje predloške sintagme (ad essa, a me) iz složene sintagme. U ovom radu nagoveštava se da suština ove pojave leži u kliticizaciji (svodjenju ad essa na ci, a me na $m i$ itd.) i da je zato treba posmatrati u širem kontekstu struktura kao Mi ci sono sdraiato "Legao sam na njega" (od: Mi sono sdraiato su di esso). Medjuzavisnost zamenica i predloga, s prilozima ili bez njih, ispoljava se na različite načine u svim evropskim jezicima, kao što se može pokazati uporedjivanjem italijanskog sa engleskim (koji nema klitike), srpskohrvatskim (koji ima naglašene i nenaglašene zamenice, ali nema "priloge-predloge" kao italijanski ), francuskim prilozima tipa dedans i nemačkim "zameničkim prilozima". 